\title{
Determinantes do envelhecimento ativo segundo a qualidade de vida e gênero
}

\author{
Determinants of active aging according to quality of life and gender
}

Ana Cristina Viana Campos ${ }^{1}$
Efigenia Ferreira e Ferreira $^{1}$
Andréa Maria Duarte Vargas $^{1}$
${ }^{1}$ Departamento de Odontologia Social e Preventiva, Faculdade de Odontologia, Universidade Federal de Minas Gerais. Av. Presidente Antônio Carlos 6627, Pampulha. 31270-010 Belo Horizonte MG Brasil. campos.acv@gmail.com

\begin{abstract}
The scope of this study was to construct an indicator of active aging and assess its association with quality of life and possible determinants according to gender. The AGEQOL (Aging, Gender and Quality of Life) study was used to interview 2052 individuals aged 60 years and older residing in Sete Lagoas in the State of Minas Gerais. The association between active aging, quality of life and possible determinants was performed by multiple logistic regression with a $5 \%$ level of statistical significance separately for each gender. Most men were in the active aging group (58\%), and $51.8 \%$ of women were in the normal aging group $(p<0.001)$. The quality of life in the Physical, Psychological, and total Score domains remained associated with the outcome in the final model for both genders. Among the men, the behavioral and community participation factors were positive predictors of active aging. Women with higher incomes, who did not suffer falls and engaged in community participation, had a better chance of belonging to the active aging group. The conclusion drawn is that quality of life and participation in groups are the main determinants of active aging, and the other factors associated with active aging are different for each gender. Key words Aging, Gender, Quality of life, Health of the elderly, Elderly
\end{abstract}

Resumo Os objetivos deste estudo foram construir um indicador de envelhecimento ativo $e$ testar sua associação com qualidade de vida e possiveis determinantes segundo gênero. O estudo AGEQOL (Aging, Gender and Quality of Life) entrevistou 2052 indivíduos com 60 anos e mais de idade residentes em Sete Lagoas, MG, Brasil. A associação entre envelhecimento ativo, qualidade de vida e possiveis determinantes foi realizada por meio da regressão logística múltipla, com nível de 5\% de significância estatística, separadamente para cada gênero. A maioria dos homens pertenceu ao grupo com envelhecimento ativo (58,0\%), enquanto $51,8 \%$ das mulheres foram alocadas no grupo com envelhecimento normal $(p<0,001)$. A qualidade de vida nos domínios Físico, Psicológico e Escore total mantiveram-se associada ao desfecho no modelo final para ambos os gêneros. Entre os homens, os fatores comportamentais e participação comunitária foram preditores positivos para envelhecimento ativo. Mulheres com maior renda, que não sofreram quedas e com participação comunitária tiveram maior chance de pertencer ao grupo com envelhecimento ativo. Conclui-se que qualidade de vida e a participação em grupos são os principais determinantes de envelhecimento ativo, e que os demais fatores determinantes associados são diferentes para cada gênero.

Palavras-chave Envelhecimento, Gênero, Qualidade de vida, Saúde do idoso, Idoso 


\section{Introdução}

O envelhecimento é um fenômeno complexo que está exigindo, cada vez mais, estudos multidisciplinares para seu melhor entendimento e compreensão. O envelhecimento ativo foi definido pela Organização Mundial de Saúde (OMS) ${ }^{1}$ como "o processo de otimização das oportunidades de saúde, participação e segurança, com o objetivo de melhorar a qualidade de vida à medida que as pessoas ficam mais velhas". De modo geral, este conceito parte do pressuposto de que envelhecer significa favorecer oportunidades para que os indivíduos possam optar por estilos de vida saudáveis e, ainda, fazer controle do próprio status de saúde e melhorar sua qualidade de vida².

De acordo também com a $\mathrm{OMS}^{3}$, qualidade de vida é a "percepção do indivíduo de sua posição na vida em relação ao contexto e sistemas de valores nos quais se insere bem como seus objetivos, expectativas, padrões e preocupações". Trata-se de um amplo conceito de classificação, afetado de modo complexo pela saúde física do indivíduo, estado psicológico, relações sociais, nível de independência e pelas suas relações com as características mais relevantes do seu meio ambiente $^{3}$. A qualidade de vida dos idosos pode ser muito boa ou, pelo menos, preservada desde que os indivíduos permaneçam ativos, com autonomia e independência, boa saúde física e relações sociais ${ }^{4}$.

Os novos estudos sobre envelhecimento apontam desafios voltados para a compreensão das condições associadas à possibilidade de assumir o envelhecimento como um processo positivo e a velhice como uma etapa da vida que pode ser acrescida de saúde, bem-estar, prazer e qualidade de vida ${ }^{5-7}$.

Portanto, os objetivos do presente estudo foram construir um indicador de envelhecimento ativo e testar sua associação com qualidade de vida e possíveis determinantes segundo gênero.

\section{Métodos}

\section{Desenho do estudo}

O presente estudo foi desenvolvido a partir de dados coletados pelo estudo AGEQOL (Aging, Gender and Quality of Life), uma coorte de base populacional sobre envelhecimento ativo, qualidade de vida e gênero realizado no município de Sete Lagoas, Minas Gerais, Brasil, em 2012. O município é dividido em 17 Regiões Administrativas
(RA), um distrito fora da sede e área rural $^{8}$ e possui 214.152 habitantes, sendo 51,4\% mulheres .

A população de referência do presente estudo foi composta por indivíduos de ambos os sexos, com idade igual ou superior a 60 anos e residentes no munícipio. Os critérios de exclusão foram: indivíduos residentes em instituição de longa permanência no período da coleta de dados, os portadores de deficiências visual e/ou auditiva graves e não corrigidas, aqueles com deficiência cognitiva que impedisse o entendimento e a execução da entrevista e do exame clínico, e os que se recusaram a assinar o Termo de Consentimento Livre e Esclarecido (TCLE).

\section{Amostragem e participantes}

O plano adotado para este estudo foi desenho complexo de amostragem. O cálculo amostral foi realizado para comparação entre os sexos considerando-se a prevalência de incapacidade funcional para atividades instrumentais entre homens $(86,6 \%)$ e mulheres $(72,9 \%)^{10}$. O erro estimado foi de até cinco pontos percentuais, poder de teste de $80 \%$, com intervalos de $95 \%$ de confiança (IC95\%), considerando um efeito de delineamento de dois. Foi utilizado um adicional de $20 \%$ para perdas e recusas. Em seguida, a amostra de cada grupo (homens e mulheres) foi estratificada por faixa etária em relação à população e corrigida pela probabilidade do risco de morrer, resultando em 1.146 idosos.

Para sorteio dos setores e domicílios, utilizou-se a técnica de amostragem probabilística por conglomerados sob o critério de partilha proporcional ao tamanho (PPT) em dois estágios: setores censitários e domicílios. Os setores e os domicílios foram sorteados proporcionalmente ao número de domicílios permanentes particulares ocupados por estrato (área urbana, zona rural, distrito). Todos os indivíduos de ambos os sexos dos domicílios selecionados com idade igual ou superior a 60 anos que concordaram em participar desta pesquisa foram entrevistados e examinados.

\section{Instrumentos e variáveis estudadas}

O instrumento era composto por informações sobre características socioeconômicas e demográficas, saneamento básico, suporte social, religiosidade, comportamentos em saúde, autopercepção de saúde, acesso e utilização de serviços de saúde ${ }^{11}$. A segunda parte do questionário continha testes e escalas de avaliação, validados 
para o português, considerando-se o conceito de envelhecimento ativo da OMS. O exame bucal foi feito sob iluminação natural, espelho bucal plano e uma espátula de madeira. $O$ índice CPOD (dentes permanentes cariados, perdidos e restaurados) foi utilizado para estimar o edentulismo, segundo critérios preconizados pela $\mathrm{OMS}^{12}$, mas adaptados.

Os indicadores utilizados como proxy de envelhecimento ativo foram: capacidade funcional, capacidade cognitiva, percepção de saúde, sintomas depressivos e funcionalidade familiar. A dependência funcional foi investigada por seis atividades básicas - AVD (tomar banho, vestir, ir ao banheiro, comer, deitar e levantar da cama/ cadeira e incontinência urinária e fecal) e oito atividades instrumentais de vida diária - AIVD (uso do telefone, usar o transporte, fazer compras, preparar uma refeição, limpar a casa, lavar a roupa, cuidar das finanças e tomar medicamentos/drogas). Os indivíduos foram classificados como independentes, parcialmente dependentes e completamente dependentes para AVD e AIVD, separadamente. Para a avaliação do estado cognitivo dos idosos, utilizou-se o Mini-Exame do Estado Mental (MEEM) ${ }^{13}$, com ponto de corte $21 / 22$ pontos $^{14}$. A presença de sintomas depressivos foi avaliada pela Escala Geriátrica de Depressão versão curta (GDS-15), com ponto de corte de 5/6. A pontuação de cinco ou mais foi considerado como indicativo de sintomas depres$\operatorname{sivos}^{15}$. O funcionamento familiar foi avaliado pelo APGAR Familiar que mensura a satisfação do idoso em relação a vários aspectos da convivência familiar. As respostas têm valores entre 1 (dificilmente) e 3 (quase sempre), de modo que a soma pode ter pontuação total de 5 a 15 . Pontuação inferior a 9 indica disfunção familiar ${ }^{16}$. A autopercepção de saúde foi avaliada por uma escala de likert para as respostas (muito ruim, ruim, regular, boa e muito boa). Para este estudo as categorias foram agrupadas em ruim (muito ruim, ruim e regular) e boa (boa e muito boa).

Para criar uma variável binária chamada "envelhecimento ativo", foi utilizada a análise de segmentação (cluster) com validação da divisão dos grupos por meio de análise discriminante dos quatro proxies de envelhecimento ativo (dependência para AVD - p $<0,001$, dependência para AIVD $-\mathrm{p}<0,001$, funcionalidade familiar $-\mathrm{p}<$ 0,001 , sintomas depressivos $-p<0,001$, déficit cognitivo $-\mathrm{p}=0,003$, percepção de saúde $-\mathrm{p}<$ 0,001 ), resultando em dois grupos (normal e ativo) - dados não apresentados. A análise de segmentação é uma ferramenta estatística utilizada para agrupar dados, alocando os casos mais similares no mesmo grupo com base nas características medidas entre os participantes do estudo ${ }^{17}$. Empregou-se o método K-Mean Cluster a fim de medir a contribuição de cada variável na formação dos clusters por meio do teste de Anova.

A análise discriminante canônica foi utilizada posteriormente para validar a análise de cluster descrita anteriormente. O objetivo da discriminação é maximizar a variância entre e dentro de grupos e verificar a eficiência da classificação correta global do modelo. Finalmente, o programa estatístico realiza uma validação interna dos resultados obtidos para verificar a eficiência de classificação entre os clusters analisados ${ }^{17}$.

A qualidade de vida foi medida pelo World Health Organization Quality of Life instrument-Bref (WHOQoL-Bref) ${ }^{18}$ e World Health Organization Quality of Life instrument-Old (WHOQoL-Old $)^{19}$. O primeiro é composto por quatro domínios e não possui escore total. O domínio Físico avalia dor e desconforto, energia e fadiga, sono e repouso, atividades da vida cotidiana, dependência de medicação ou de tratamentos, capacidade de trabalho. O domínio Psicológico envolve questões sobre sentimentos positivos, memória e concentração, autoestima, imagem corporal e aparência, sentimentos negativos e espiritualidade, religiosidade, crenças pessoais. As relações pessoais, suporte (apoio) social e atividade sexual compõem o domínio das Relações Sociais. O último domínio - Meio ambiente - é o maior e mede segurança física e proteção, ambiente no lar, recursos financeiros, cuidados de saúde e sociais, oportunidades de adquirir novas informações e habilidades, oportunidades de recreação/lazer, ambiente físico e transporte ${ }^{18}$. O módulo WHOQoL-Old consiste em 24 itens da escala de likert atribuídos a seis facetas: "Funcionamento do Sensório" (FS), “Autonomia” (AUT), "Atividades Passadas, Presentes e Futuras" (PPF), "Participação Social" (PSO), "Morte e Morrer" (MEM) e "Intimidade" (INT). Os escores destas seis facetas foram combinados para produzir um escore total para a qualidade de vida em idosos ${ }^{19}$.

Para conhecer mais detalhadamente a expressão dos determinantes do envelhecimento ativo na vida dos idosos, as variáveis independentes foram agrupadas em um modelo multidimensional elaborado a partir dos determinantes propostos pela $\mathrm{OMS}^{20,21}$ : fatores comportamentais, pessoais, ambiente físico, ambiente social, econômico, sistemas de saúde e serviço social e fatores transversais (cultura e gênero). Os fatores transversais foram avaliados por naturalidade (Sete Lagoas, 
outra cidade mineira, outro estado), faixa etária (60-74 anos, $\geq 75$ anos), cor da pele (branca, preta/parda, outras), estado civil (casado, separado/ solteiro/viúvo), religião (católica, evangélica, espírita, outra, sem religião). Os fatores relacionados aos sistemas de saúde foram: consulta médica (sim, não), plano de saúde (sim, não), serviço de saúde mais utilizado (público, plano/convênio, particular). Os hábitos e o estilo de vida forma agrupados em fatores comportamentais: fumo (sim, não), álcool (sim, não), atividade física (sim, não), uso de medicamento (sim, não), perda de apetite (sim, não), edentulismo (sim, não). Os fatores pessoais foram avaliados pela satisfação com a vida (feliz, nem feliz nem infeliz, infeliz). Em relação aos fatores relacionados ao ambiente físico investigaram-se: local de moradia: (área urbana, rural/distrito), posse do domicílio (próprio, alugado, de parentes, outro), tipo de construção do domicílio (concreto, outros) esgoto canalizado (sim, não), abastecimento de água canalizado (sim, não), sanitário no domicílio (sim, não), coleta de lixo (sim, não), eletricidade (sim, não), quedas nos últimos seis meses anteriores à entrevista (sim, não). O ambiente social foi investigado por meio de: nível de escolaridade (analfabeto, $1^{\circ}$ grau, $2^{\circ}$ grau), tipo de arranjo domiciliar (mora com cônjuge, arranjos mistos/ intergeracionais, mora sozinho), participação em grupos formais ou informais (nenhum; $\geq$ 01 ), número de amigos (nenhum, 1-10, $\geq 11$ ), confiança nas pessoas (sim; não), sentimento de segurança quando está sozinho em casa (seguro; nem seguro nem inseguro; inseguro). A renda mensal dicotomizada pela mediana $(\leq \mathrm{R} \$ 622,00$; $>\mathrm{R} \$ 622,00)$ e aposentado (sim, não) foram os fatores econômicos investigados.

\section{Estudo piloto e coleta de dados}

O projeto piloto foi conduzido anteriormente à coleta de dados com 107 idosos $(10 \%$ da amostra total) em um município vizinho. Apesar dos instrumentos serem validados para o Brasil, para verificação da confiabilidade e desempenho dos questionários e testes nesta população, o método teste/reteste foi empregado. Obtiveram-se coeficientes superiores a $0,80(\mathrm{p}<0,001)$; Kappa Ponderado (IC $95 \%)=0,81(0,71-0,91)$; Kappa ajustado $=0,86$.

A coleta de dados foi realizada no domicílio do idoso entre os meses de janeiro e julho de 2012 por três examinadores e por três anotadores calibrados. As entrevistas duravam de 40 a 60 minutos. Foram consideradas perdas de entrevista/ exame os casos em que o idoso não foi localizado no domicilio após três tentativas, incluindo fim de semana. $\mathrm{O}$ projeto foi divulgado junto às autoridades municipais, meios de comunicação e por meio de folder próprio.

O projeto foi aprovado pelo Comitê de Ética em Pesquisa da Universidade Federal de Minas Gerais.

\section{Análise dos dados}

Para o presente artigo foram selecionadas análises de frequências entre os sexos mediante testes chi-quadrado, com margem de erro aceitável de $5 \%$. Todas as informações sobre as variáveis do estudo foram armazenadas em um banco de dados do Statistical Package for Social Sciences (SPSS) para Windows, versão 19.0 e a análise dos dados se deu em duas fases. Na primeira, foram descritas as variáveis em relação ao envelhecimento ativo, calculando-se frequências relativas e absolutas. Para os escores do WHOQOL-bref e WHOQOL-old foram calculadas médias e desvio-padrão. Em seguida, foi feita a avaliação por bloco das variáveis em estudo, por meio da regressão logística múltipla, de natureza exploratória, pelo método backward. As variáveis que alcançaram significância estatística inferior a $0,25(\mathrm{p}<0,25)^{22}$, na primeira fase, foram incluídas no modelo saturado. O modelo final ajustado conservou apenas as variáveis que atingiram o nível de 5\% de significância estatística $(\mathrm{p}<0,05)$.

Todas as análises foram realizadas para homens e mulheres separadamente. Os testes de homogeneidade dos declives e de multicolinearidade foram realizados com ajuste de Pearson para analisar a validade do modelo construído. Para verificar a possível interferência do número reduzido de algumas observações, utilizou-se a análise dos resíduos de Pregibon ${ }^{23}$. O efeito do desenho amostral complexo foi considerado em todas as análises realizadas.

\section{Resultados}

Entre os domicílios sorteados para obter a amostra, 27 idosos haviam mudado de endereço, 38 não foram localizados e 35 faleceram no período de coleta de dados, totalizando uma perda de $4,8 \%$. Entre os idosos encontrados nos domicílios, houve $5,8 \%$ de recusa e $1,2 \%$ de perdas por outros motivos. Foram realizadas 25 entrevistas com o cuidador do idoso, devido a dificuldades físicas e/ou mentais, não tendo sido, por esse 
motivo, incluídas no estudo. Portanto, a amostra final foi constituída por 2.052 indivíduos, sendo $59,7 \%$ do sexo feminino.

A idade da amostra total no início do estudo variou entre 60 e 106 anos. A idade média foi de $70,9 \pm 8,1$ anos $(71,3 \pm 8,3$ anos para as mulheres e de 70,7 \pm 7,8 anos para os homens).

$\mathrm{Na}$ Tabela 1 se encontram as estatísticas descritivas dos fatores transversais, comportamentais, pessoais, ambiente físico, ambiente social, econômico, sistemas de saúde e serviço social dos participantes, além das análises de significação estatística entre os sexos. A maioria dos homens $(70,8 \%)$ e mulheres $(68,7 \%)$ tinha entre 60 e 74 anos de idade. Não houve diferença na distribuição etária entre os diferentes sexos.

Não houve diferenças entre os sexos em relação à escolaridade. No entanto, 10,4\% dos homens e $8,6 \%$ das mulheres cursaram o segundo grau (Tabela 1).

Além disso, observou-se que houve diferença estatisticamente significante entre os sexos relacionados ao estado civil, consulta médica, plano de saúde, fumo, álcool, medicamento, perda de apetite, edentulismo, satisfação com a vida, tipo de domicilio, esgoto canalizado, eletricidade, arranjo domiciliar, percepção da segurança, renda mensal e aposentadoria (Tabela 1).

Foram identificados quarenta e oito tipos de condições de vida entre idosos no município em estudo. Levando-se em conta os três grupos de condições de vida estabelecidos neste estudo, observou-se que a maioria dos idosos que vivem sozinhos são mulheres $(71,5 \%)$, enquanto $75,5 \%$ dos homens viviam com seus cônjuges $(\mathrm{p}<0,001)$. A maioria dos homens na amostra era casada $(74,5 \%)$, enquanto $61,7 \%$ das mulheres eram solteiras, separadas ou viúvas. A maioria dos idosos tem baixa renda mensal $(66,1 \%)$, sendo esse percentual maior entre as mulheres $(71,5 \%)$ em comparação com o sexo masculino $(58,1 \%)$ (Tabela 1$)$.

A maioria dos idosos $(\mathrm{n}=1070)$ pertenceu ao grupo de envelhecimento ativo ao passo que $982(47,85 \%)$ idosos representaram o grupo com envelhecimento normal. $\mathrm{Na}$ análise de sexo, observou-se que a maioria homens pertenceu ao grupo com envelhecimento ativo $(58,0 \%)$, enquanto $51,8 \%$ das mulheres foram alocadas no grupo com envelhecimento normal ( $\mathrm{p}<0,001)$.

Nas Tabelas 2 e 3 podem ser observados os indicadores de associação entre envelhecimento ativo, os domínios da qualidade de vida e fatores determinantes para os sexos feminino e masculino, respectivamente. $\mathrm{Na}$ análise da odds ratio bruto, observou-se uma forte associação do envelhecimento ativo com Domínio Físico (OR = 1,07 para homens e $\mathrm{OR}=1,05$ para mulheres) e Escore total do WHOQOL-old ( $\mathrm{OR}=1,03$ para homens e $\mathrm{OR}=1,03$ para mulheres). Os homens do grupo com envelhecimento ativo tiveram 1,03 (IC95\%:1,01-1,05) e 1,02 (IC95\%:1,00-1,04) mais chances de ter maiores médias no Domínio Psicológico e Ambiental, respectivamente, em comparação com seus pares com envelhecimento normal. O Domínio Psicológico também esteve diretamente associado com envelhecimento ativo entre as idosas $(\mathrm{OR}=1,01, \mathrm{p}=0,023)$.

$\mathrm{Na}$ análise bruta, observou-se que os fatores comportamentais (atividade física, medicamento, perda de apetite) se associaram significativamente com envelhecimento ativo para o sexo feminino (Tabela 2) e masculino (Tabela 3). Para ambos os sexos, também houve associação estatística entre envelhecimento ativo, quedas (OR $=0,51$ para o sexo feminino e $\mathrm{OR}=0,55$ para o masculino), percepção de felicidade $(\mathrm{OR}=0,51$ para o sexo feminino e $\mathrm{OR}=0,55$ para o masculino), analfabetismo ( $\mathrm{OR}=0,54$ para o sexo feminino e $\mathrm{OR}=0,43$ para o masculino) e $1^{\circ}$ grau escolar $(\mathrm{OR}=0,48$ para o sexo feminino e $\mathrm{OR}=$ 0,42 para o masculino)

Para o sexo feminino, os fatores relacionados aos sistemas de saúde se associaram inversamente com o desfecho. Mulheres que tiveram consulta médica nos últimos seis meses $(\mathrm{OR}=0,42)$, possuem plano de saúde $(\mathrm{OR}=0,68)$ e consultaram o serviço público de saúde $(\mathrm{OR}=0,67)$ tiveram maior chance de pertencerem ao grupo com envelhecimento normal (Tabela 2).

Homens fumantes e aposentados tiveram 0,64 (IC95\%:0,43-0,95) e 0,64 (IC95\%:0,43$0,95)$ menos chance de ter envelhecimento ativo, quando comparados aos homens que não fumavam e aos não aposentados, respectivamente (Tabela 3).

Os resultados do modelo final de regressão logística para estimar OR de envelhecimento ativo para o sexo masculino e feminino são mostrados na Tabela 4. Para ambos os sexos, o envelhecimento ativo foi explicado pela qualidade de vida, fatores comportamentais, fatores relacionados ao ambiente social e econômico.

A qualidade de vida nos domínios Físico, Psicológico e Escore total manteve-se associada ao desfecho no modelo final para ambos os sexos. Maiores escores no domínio físico aumentaram em 1,07 (OR = 1,07; p = 0,000) e 1,05 (OR = 1,05; $\mathrm{p}=0,000)$ as chances de envelhecimento ativo para homens e mulheres, respectivamente (Tabela 4). 
Tabela 1. Fatores transversais, comportamentais, pessoais, ambiente físico, ambiente social, econômico, sistemas de saúde e serviço social de idosos residentes em comunidade de Sete Lagoas, Minas Gerais, Brasil em função do gênero, 2012.

\begin{tabular}{|c|c|c|c|c|c|}
\hline & \multicolumn{2}{|c|}{ Masculino } & \multicolumn{2}{|c|}{ Feminino } & \multirow[b]{2}{*}{ p-valor } \\
\hline & $\mathbf{n}$ & $\%$ & $\mathbf{n}$ & $\%$ & \\
\hline \multicolumn{6}{|l|}{ Fatores transversais } \\
\hline Naturalidade & & & & & 0,859 \\
\hline Sete Lagoas & 235 & 28,5 & 362 & 29,6 & \\
\hline Outra cidade mineira & 561 & 67,9 & 818 & 66,8 & \\
\hline Outro estado & 30 & 3,6 & 45 & 3,7 & \\
\hline Faixa etária & & & & & 0,305 \\
\hline $60-74$ anos & 585 & 70,8 & 842 & 68,7 & \\
\hline$\geq 75$ anos & 241 & 29,2 & 384 & 31,3 & \\
\hline Cor da pele & & & & & 0,585 \\
\hline Branca & 298 & 36,4 & 421 & 34,7 & \\
\hline Preta/Parda & 132 & 16,1 & 188 & 15,5 & \\
\hline Outras & 389 & 47,5 & 605 & 49,8 & \\
\hline Estado civil & & & & & $<0,001$ \\
\hline Casado & 615 & 74,5 & 469 & 38,3 & \\
\hline Separado/solteiro/viúvo & 211 & 25,5 & 754 & 61,7 & \\
\hline Religião & & & & & $<0,001$ \\
\hline Católica & 664 & 80,4 & 925 & 75,4 & \\
\hline Evangélica & 115 & 13,9 & 263 & 21,5 & \\
\hline Espírita & 13 & 1,6 & 09 & 0,7 & \\
\hline Outra & 06 & 0,7 & 16 & 1,3 & \\
\hline Sem religião & 28 & 3,4 & 13 & 1,1 & \\
\hline \multicolumn{6}{|c|}{ Fatores relacionados aos sistemas de saúde } \\
\hline Consulta médica & & & & & $<0,001$ \\
\hline Sim & 700 & 84,7 & 1107 & 90,3 & \\
\hline Não & 126 & 15,3 & 119 & 9,7 & \\
\hline Plano de saúde & & & & & 0,012 \\
\hline Sim & 466 & 56,4 & 761 & 62,1 & \\
\hline Não & 360 & 43,6 & 465 & 37,9 & \\
\hline Serviço de saúde & & & & & 0,261 \\
\hline Público & 477 & 57,7 & 676 & 55,1 & \\
\hline Plano/Convênio & 242 & 29,3 & 401 & 32,7 & \\
\hline Particular & 107 & 13,0 & 149 & 12,2 & \\
\hline \multicolumn{6}{|l|}{ Fatores comportamentais } \\
\hline Fumo & & & & & $<0,001$ \\
\hline Sim & 143 & 17,3 & 82 & 6,7 & \\
\hline Não & 683 & 82,7 & 1144 & 93,3 & \\
\hline Álcool & & & & & $<0,001$ \\
\hline Sim & 257 & 31,1 & 126 & 10,3 & \\
\hline Não & 569 & 68,9 & 1100 & 89,7 & \\
\hline Atividade física & & & & & 0,760 \\
\hline Sim & 216 & 26,2 & 329 & 26,8 & \\
\hline Não & 610 & 73,8 & 897 & 73,2 & \\
\hline Medicamento & & & & & $<0,001$ \\
\hline Sim & 579 & 70,1 & 973 & 79,4 & \\
\hline Não & 247 & 29,9 & 253 & 20,6 & \\
\hline Perda de apetite & & & & & $<0,001$ \\
\hline Sim & 179 & 21,7 & 390 & 31,8 & \\
\hline Não & 647 & 78,3 & 836 & 68,2 & \\
\hline Edentulismo & & & & & 0,008 \\
\hline Sim & 520 & 63,0 & 843 & 68,8 & \\
\hline Não & 305 & 37,0 & 382 & 31,2 & \\
\hline
\end{tabular}




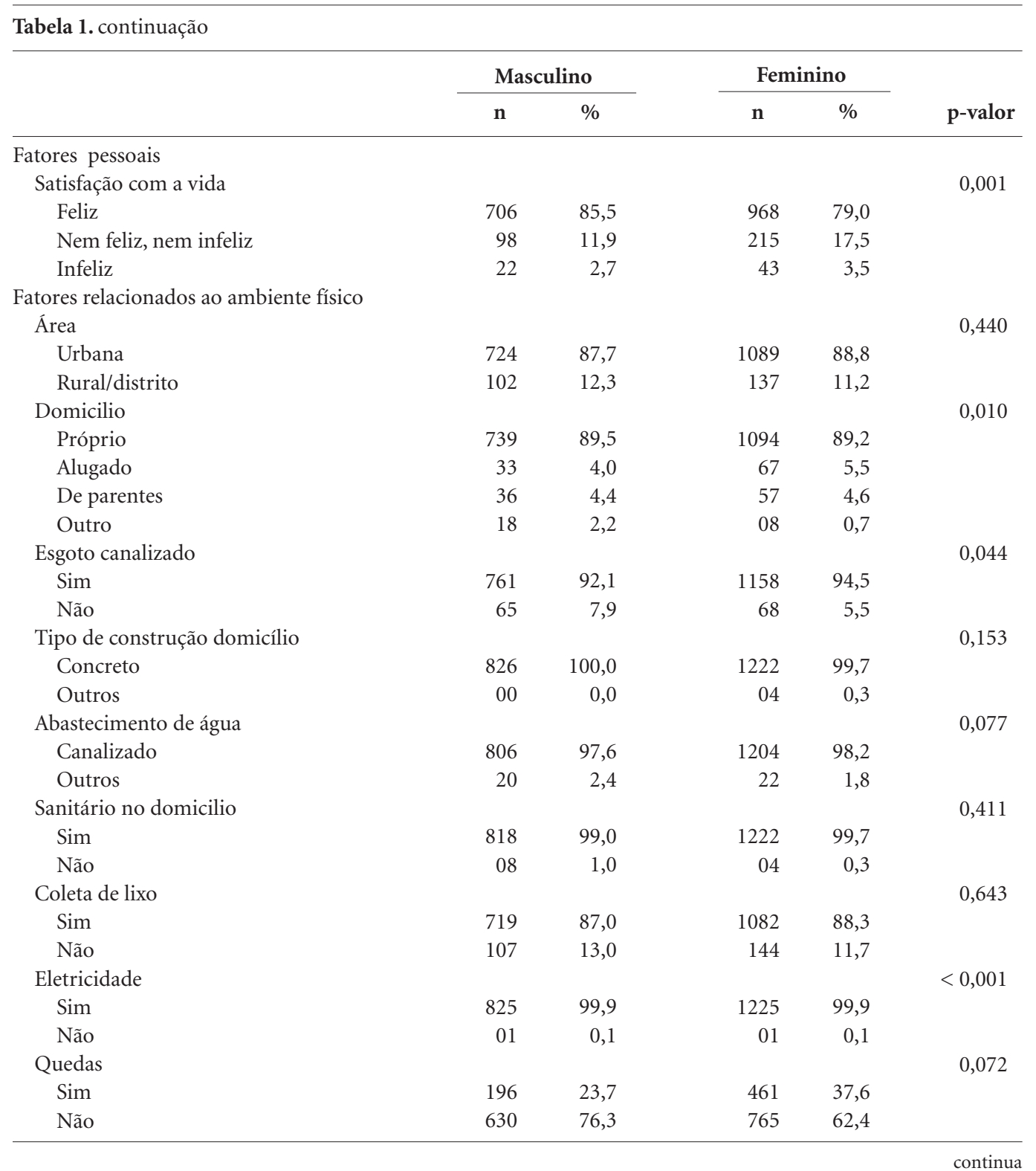

Para ambos os sexos, houve uma forte associação entre envelhecimento ativo e participação em grupos. A participação em grupos aumentou em $88 \%(\mathrm{OR}=1,88)$ e $65 \%(\mathrm{OR}=1,65)$ a chance de envelhecimento ativo para homens e mulheres, respectivamente (Tabela 4).

Entre os homens, observou-se uma associação significativa entre envelhecimento ativo e atividade física ( $\mathrm{OR}=1,63 ; \mathrm{p}=0,008)$, ou seja, envelhecimento ativo foi de 1,63 vezes superior entre os homens que praticavam atividade física quando comparados aos idosos sedentários (Tabela 4$)$.
Perda de apetite em ambos os sexos, aposentadoria entre os homens e baixa renda mensal entre as mulheres foram fatores de risco para envelhecimento normal. Homens que fumavam $(\mathrm{OR}=0,53$; IC95\%:0,34-0,82) e mulheres que sofreram queda (OR = 0,64; IC95\%:0,49-0,84) tiveram menor chance de ter um envelhecimento ativo quando comparadas aos homens não fumantes e às mulheres que não sofreram queda, respectivamente (Tabela 4). 


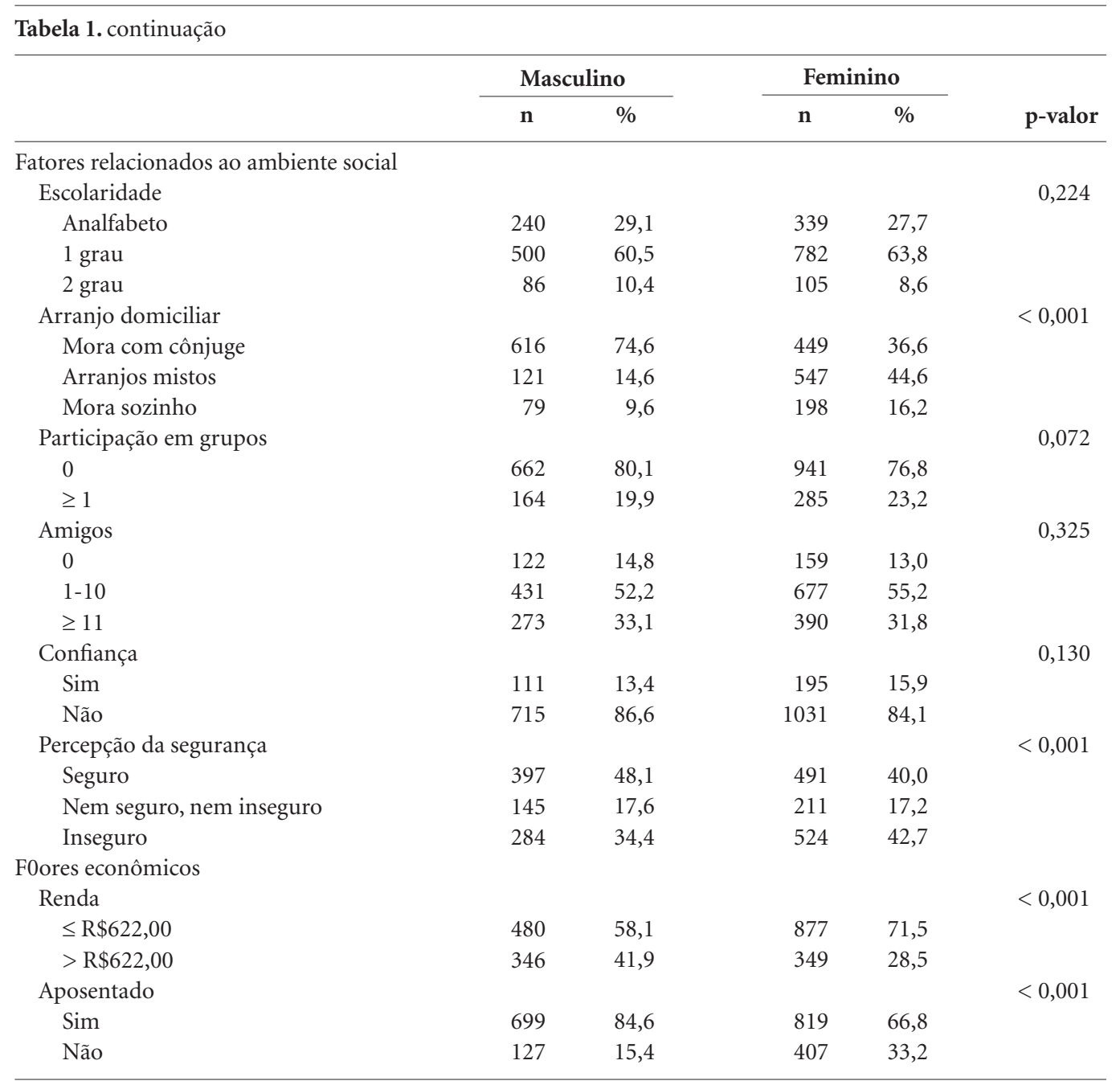

\section{Discussão}

Independência funcional, capacidade cognitiva, depressão, percepção de saúde e funcionamento familiar representam fatores importantes para a ação política do envelhecimento ativo ${ }^{24-27}$, porque eles têm o potencial de afetar a saúde e a qualidade de vida de idosos. Sendo assim, assumimos o desafio de construir um indicador multidimensional de envelhecimento ativo unindo todas essas variáveis e testar a influência de determinantes na associação com qualidade de vida.

$\mathrm{O}$ envelhecimento ativo é diferente entre os gêneros, sendo que $64,7 \%$ dos idosos do grupo desfavorável são mulheres. Esses resultados indicam que as mulheres possuem maiores taxas de dependência funcional, déficit cognitivo, depressão, pior funcionamento familiar e uma percepção negativa da própria saúde quando comparadas aos homens. Uma possível explicação é alta prevalência de dependência funcional entre as mulheres $(33,0 \%)$ quando comparada aos homens $(23,7 \%)$, bem como alta prevalência de depressão (34,4\% no sexo feminino e $23,8 \%$ no sexo masculino).

As diferenças de gênero estão relacionadas aos padrões socioculturais do comportamento humano e são fatores relevantes que determinam a ocorrência de eventos e atitudes que podem se tornar limites ou possibilidades para a conquista do envelhecimento mais ativo e com qualidade de vida ${ }^{28,29}$.

Este estudo é original e inovador, pois apresenta informações a respeito de uma associação positiva entre envelhecimento ativo e qualidade de vida em uma amostra representativa de idosos para ambos os gêneros. Além disso, nossos resultados indicam que os fatores mais importantes para o envelhecimento ativo para ambos os gêneros é melhor qualidade de vida, participar 
Tabela 2. Fatores associados ao envelhecimento ativo entre mulheres idosas residentes em comunidade. Sete lagoas-MG, 2012.

\begin{tabular}{|c|c|c|c|c|c|c|}
\hline \multirow{3}{*}{ Fatores determinantes } & \multicolumn{4}{|c|}{ Envelhecimento } & \multirow{3}{*}{$\begin{array}{l}\text { OR bruto } \\
\text { (IC95\%) }\end{array}$} & \multirow{3}{*}{ p-valor } \\
\hline & \multicolumn{2}{|c|}{ Normal $(\mathrm{N}=635)$} & \multicolumn{2}{|c|}{ Ativo $(\mathrm{N}=591)$} & & \\
\hline & $\mathbf{n}$ & $\%$ & $\mathbf{n}$ & $\%$ & & \\
\hline \multicolumn{7}{|l|}{ Qualidade de vida } \\
\hline Domínio Físico* & $53,80( \pm 16,86)$ & & $68,78( \pm 14,32)$ & & $1,05(1,04-1,06)$ & 0,000 \\
\hline Domínio Psicológico* & $61,90( \pm 15,71)$ & & $71,96( \pm 11,88)$ & & $1,01(1,00-1,03)$ & 0,023 \\
\hline Domínio Relações Sociais* & $65,93( \pm 15,54)$ & & $70,97( \pm 14,04)$ & & $0,99(0,98-1,00)$ & 0,202 \\
\hline Domínio Ambiental $^{*}$ & $56,68( \pm 12,77)$ & & $64,19( \pm 12,17)$ & & $1,01(0,99-1,02)$ & 0,248 \\
\hline Escore total $^{*}$ & $61,90( \pm 11,27)$ & & $70,23( \pm 10,99)$ & & $1,03(1,01-1,04)$ & 0,001 \\
\hline \multicolumn{7}{|l|}{ Fatores transversais } \\
\hline \multicolumn{7}{|l|}{ Naturalidade } \\
\hline Sete Lagoas & 181 & 28,5 & 181 & 30,6 & $1,66(0,87-3,19)$ & 0,125 \\
\hline Outra cidade mineira & 424 & 66,9 & 394 & 66,7 & $1,56(0,83-2,93)$ & 0,168 \\
\hline Outro estado & 29 & 4,6 & 16 & 2,7 & 1 & \\
\hline \multicolumn{7}{|l|}{ Faixa etária } \\
\hline $60-74$ anos & 434 & 68,3 & 408 & 69,0 & $1,03(0,80-1,33)$ & 0,794 \\
\hline$\geq 75$ anos & 201 & 31,7 & 183 & 31,0 & 1 & \\
\hline \multicolumn{7}{|l|}{ Cor da pele } \\
\hline Branca & 213 & 33,8 & 208 & 35,7 & $1,03(0,80-1,32)$ & 0,842 \\
\hline Preta/Parda & 103 & 16,3 & 85 & 14,6 & $0,90(0,65-1,26)$ & 0,546 \\
\hline Outras & 315 & 49,9 & 290 & 49,7 & 1 & \\
\hline \multicolumn{7}{|l|}{ Estado civil } \\
\hline Casado & 234 & 37,0 & 235 & 39,8 & $1,12(0,88-1,42)$ & 0,361 \\
\hline Separado/solteiro/viúvo & 399 & 63,0 & 355 & 60,2 & 1 & \\
\hline \multicolumn{7}{|l|}{ Religião } \\
\hline Católica & 466 & 73,4 & 459 & 77,7 & $1,91(0,57-6,41)$ & 0,297 \\
\hline Evangélica & 147 & 23,1 & 116 & 19,6 & $1,54(0,45-5,26)$ & 0,495 \\
\hline Espírita & 04 & 0,6 & 05 & 0,8 & $2,44(0,41-14,54)$ & 0,327 \\
\hline Outra & 10 & 1,6 & 06 & 1,0 & $1,30(0,27-6,32)$ & 0,744 \\
\hline Sem religião & 08 & 1,3 & 05 & 0,8 & 1 & \\
\hline \multicolumn{7}{|c|}{ Fatores relacionados aos Sistemas de Saúde } \\
\hline \multicolumn{7}{|l|}{ Consulta médica } \\
\hline $\operatorname{Sim}$ & 597 & 94,0 & 510 & 86,3 & $0,42(0,28-0,64)$ & 0,000 \\
\hline Não & 38 & 6,0 & 81 & 13,7 & 1 & \\
\hline \multicolumn{7}{|l|}{ Plano de saúde } \\
\hline $\operatorname{Sim}$ & 395 & 62,2 & 366 & 61,9 & $0,68(0,51-0,90)$ & 0,008 \\
\hline Não & 240 & 37,8 & 225 & 38,1 & 1 & \\
\hline \multicolumn{7}{|l|}{ Serviço de saúde } \\
\hline Público & 384 & 60,5 & 292 & 49,4 & $0,67(0,46-0,97)$ & 0,038 \\
\hline Plano/Convênio & 179 & 28,2 & 222 & 37,6 & $1,34(0,90-1,98)$ & 0,141 \\
\hline Particular & 72 & 11,3 & 77 & 13,0 & 1 & \\
\hline
\end{tabular}

de grupos comunitários e não ter relatado perda de apetite, mesmo quando o modelo foi ajustado para as condições socioeconômicas.

Estudos mostram que o envelhecimento ativo está relacionado à maior longevidade dos idosos, boa participação social, melhorias nas condições de saúde e cuidado, com manutenção ou melhoria da qualidade de vida ${ }^{20,21}$.

Nossos resultados e outros estudos ${ }^{30,31}$, corroboram a qualidade de vida como preditor mais forte para envelhecimento ativo nesta amostra de idosos,. Neste estudo, a associação entre envelhe- 
Tabela 2. continuação

Envelhecimento

\begin{tabular}{|c|c|c|c|c|c|c|}
\hline \multirow{3}{*}{ Fatores determinantes } & \multirow{2}{*}{\multicolumn{2}{|c|}{ Normal $(\mathrm{N}=635)$}} & \multirow{2}{*}{\multicolumn{2}{|c|}{ Ativo $(\mathrm{N}=591)$}} & \multirow{3}{*}{$\begin{array}{c}\text { OR bruto } \\
\text { (IC95\%) }\end{array}$} & \multirow{3}{*}{ p-valor } \\
\hline & & & & & & \\
\hline & n & $\%$ & $\mathbf{n}$ & $\%$ & & \\
\hline \multicolumn{7}{|l|}{ Fatores comportamentais } \\
\hline Fumo & 43 & 6,8 & 39 & 6,6 & $0,92(0,58-1,59)$ & 0,761 \\
\hline Sim & 592 & 93,2 & 552 & 93,4 & 1 & \\
\hline \multicolumn{7}{|l|}{ Não } \\
\hline Álcool & 60 & 9,4 & 66 & 11,2 & $0,99(0,67-1,47)$ & 0,966 \\
\hline Sim & 575 & 90,6 & 525 & 88,8 & 1 & \\
\hline \multicolumn{7}{|l|}{ Não } \\
\hline Atividade física & 142 & 22,4 & 187 & 31,6 & $1,42(1,08-1,85)$ & 0,011 \\
\hline Sim & 493 & 77,6 & 404 & 68,4 & 1 & \\
\hline \multicolumn{7}{|l|}{ Não } \\
\hline Medicamento & 541 & 85,2 & 432 & 73,1 & $0,50(0,37-0,67)$ & 0,000 \\
\hline Sim & 94 & 14,8 & 159 & 26,9 & 1 & \\
\hline \multicolumn{7}{|l|}{ Não } \\
\hline Perda de apetite & 265 & 41,7 & 125 & 21,2 & $0,40(0,31-0,52)$ & 0,000 \\
\hline Sim & 370 & 58,3 & 466 & 78,8 & 1 & \\
\hline \multicolumn{7}{|l|}{ Não } \\
\hline Edentulismo & 456 & 71,8 & 387 & 65,6 & $0,82(0,63-1,05)$ & 0,116 \\
\hline Sim & 179 & 28,2 & 203 & 34,4 & 1 & \\
\hline Não & & & & & & \\
\hline \multicolumn{7}{|l|}{ Fatores pessoais } \\
\hline \multicolumn{7}{|l|}{ Percepção de felicidade } \\
\hline Feliz & 453 & 71,3 & 515 & 87,1 & $2,36(1,23-4,50)$ & 0,010 \\
\hline Nem feliz, nem infeliz & 153 & 24,1 & 62 & 10,5 & $0,84(0,42-1,69)$ & 0,625 \\
\hline Infeliz & 29 & 4,6 & 14 & 2,4 & 1 & \\
\hline \multicolumn{7}{|c|}{ Fatores relacionados ao ambiente físico } \\
\hline \multicolumn{7}{|c|}{ Área } \\
\hline Urbana & 558 & 87,9 & 531 & 89,8 & $0,80(0,49-1,32)$ & 0,389 \\
\hline Rural/distrito & 77 & 12,1 & 60 & 10,2 & 1 & \\
\hline \multicolumn{7}{|l|}{ Domicílio } \\
\hline Próprio & 566 & 89,1 & 528 & 89,3 & $1,04(0,24-4,43)$ & 0,959 \\
\hline Alugado & 35 & 5,5 & 32 & 5,4 & $0,98(0,21-4,54)$ & 0,984 \\
\hline De parentes & 30 & 4,7 & 27 & 4,6 & $1,09(0,23-5,06)$ & 0,916 \\
\hline Outro & 04 & 0,6 & 04 & 0,7 & 1 & \\
\hline \multicolumn{7}{|l|}{ Esgoto canalizado } \\
\hline Sim & 592 & 93,2 & 566 & 95,8 & $2,24(1,08-4,63)$ & 0,030 \\
\hline Não & 43 & 6,8 & 25 & 4,2 & 1 & \\
\hline Tipo de construção domicílio & & & & & & \\
\hline Concreto & 634 & 99,8 & 588 & 99,5 & $0,27(0,02-3,45)$ & 0,317 \\
\hline Outros & 01 & 0,2 & 03 & 0,5 & 1 & \\
\hline Abastecimento de água & & & & & & \\
\hline Canalizado & 622 & 98,0 & 582 & 98,5 & $0,95(0,32-2,81)$ & 0,928 \\
\hline Outros & 13 & 2,0 & 09 & 1,5 & 1 & \\
\hline Sanitário no domicílio & & & & & & \\
\hline $\operatorname{Sim}$ & 634 & 99,8 & 588 & 99,5 & $0,26(0,02-3,42)$ & 0,308 \\
\hline Não & 01 & 0,2 & 03 & 0,5 & 1 & \\
\hline Coleta de lixo & & & & & & \\
\hline Sim & 558 & 87,9 & 524 & 88,7 & $1,04(0,70-1,55)$ & 0,850 \\
\hline Não & 77 & 12,1 & 67 & 11,3 & 1 & \\
\hline Eletricidade & & & & & & \\
\hline Sim & 635 & 100,0 & 590 & 99,8 & $0,00(0,00-0,00)$ & 1,000 \\
\hline Não & 00 & 0,0 & 01 & 0,2 & 1 & \\
\hline Quedas & & & & & & \\
\hline Sim & 286 & 45,0 & 175 & 29,6 & $0,51(0,40-0,64)$ & 0,000 \\
\hline Não & 349 & 55,0 & 416 & 70,4 & 1 & \\
\hline
\end{tabular}




\begin{tabular}{|c|c|c|c|c|c|c|}
\hline \multirow{3}{*}{ Fatores determinantes } & \multicolumn{4}{|c|}{ Envelhecimento } & \multirow{3}{*}{$\begin{array}{l}\text { OR bruto } \\
\text { (IC95\%) }\end{array}$} & \multirow{3}{*}{ p-valor } \\
\hline & \multicolumn{2}{|c|}{ Normal $(\mathrm{N}=635)$} & \multicolumn{2}{|c|}{ Ativo $(\mathrm{N}=591)$} & & \\
\hline & $\mathbf{n}$ & $\%$ & $\mathbf{n}$ & $\%$ & & \\
\hline \multirow{2}{*}{\multicolumn{7}{|c|}{$\begin{array}{l}\text { Fatores relacionados ao ambiente social } \\
\text { Escolaridade }\end{array}$}} \\
\hline & & & & & & \\
\hline Analfabeto & 188 & 29,6 & 151 & 25,5 & $0,54(0,35-0,83)$ & 0,005 \\
\hline 1 grau & 408 & 64,3 & 374 & 63,3 & $0,48(0,30-0,77)$ & 0,002 \\
\hline 2 grau & 39 & 6,1 & 66 & 11,2 & 1 & \\
\hline \multicolumn{7}{|l|}{ Arranjo domiciliar } \\
\hline Mora com cônjuge & 227 & 36,4 & 222 & 38,9 & $1,16(0,83-1,64)$ & 0,381 \\
\hline Arranjos mistos & 290 & 46,5 & 257 & 45,1 & $1,07(0,76-1,49)$ & 0,704 \\
\hline Mora sozinho & 107 & 17,1 & 91 & 16,0 & 1 & \\
\hline \multicolumn{7}{|l|}{ Participação em grupos } \\
\hline 0 & 486 & 76,5 & 455 & 77,0 & $1,10(0,84-1,46)$ & 0,481 \\
\hline$\geq 1$ & 149 & 23,5 & 136 & 23,0 & 1 & \\
\hline \multicolumn{7}{|l|}{ Amigos } \\
\hline 0 & 88 & 13,9 & 71 & 12,0 & $0,75(0,51-1,10)$ & 0,141 \\
\hline $1-10$ & 367 & 57,8 & 310 & 52,5 & $0,72(0,56-0,93)$ & 0,013 \\
\hline$\geq 11$ & 180 & 28,3 & 210 & 35,5 & 1 & \\
\hline \multicolumn{7}{|l|}{ Confiança } \\
\hline Sim & 95 & 15,0 & 100 & 16,9 & $0,93(0,67-1,30)$ & 0,672 \\
\hline Não & 540 & 85,0 & 491 & 83,1 & & \\
\hline \multicolumn{7}{|l|}{ Percepção da segurança } \\
\hline Seguro & 222 & 35,0 & 269 & 45,5 & $1,67(1,28-2,18)$ & 0,000 \\
\hline Nem seguro, nem inseguro & 107 & 16,9 & 104 & 17,6 & $1,30(0,93-1,81)$ & 0,119 \\
\hline Inseguro & 306 & 48,2 & 218 & 36,9 & 1 & \\
\hline \multicolumn{7}{|l|}{ Fatores econômicos } \\
\hline \multicolumn{7}{|l|}{ Renda } \\
\hline$\leq \mathrm{R} \$ 622,00$ & 483 & 76,1 & 394 & 66,7 & $0,63(0,49-0,81)$ & 0,000 \\
\hline$>\mathrm{R} \$ 622,00$ & 152 & 23,9 & 197 & 33,3 & 1 & \\
\hline \multicolumn{7}{|l|}{ Aposentado } \\
\hline Sim & 417 & 65,7 & 402 & 68,0 & $1,07(0,84-1,36)$ & 0,605 \\
\hline Não & 218 & 34,3 & 189 & 32,0 & 1 & \\
\hline
\end{tabular}

*Valores expressados em média ( \pm desvio padrão).

cimento ativo e qualidade de vida para ambos os gêneros se manteve fortemente significante, mesmo depois do ajuste do modelo por todas as outras variáveis; embora a direção de causa e efeito não possa ser confirmada.

Outros estudos realizados no Brasi ${ }^{32}$ e em outros países ${ }^{33,34}$ mostram que há uma relação de impacto entre gênero, qualidade de vida sobre o envelhecimento, que merece ser mais bem investigada.

Observou-se também uma relação diretamente proporcional entre envelhecimento ativo e participação em grupos, ou seja, quanto maior a participação comunitária dos idosos, maior a chance de envelhecimento ativo.

Uma rede social reduzida pode contribuir para exacerbar incapacidades ou impor limita- ções no estilo de vida, contribuindo para o isolamento social, que por sua vez, pode interferir na saúde e na qualidade de vida do idoso ${ }^{35}$.

Por outro lado, a participação comunitária dos idosos deste estudo é muito baixa (21,9\%), sendo que a maioria das mulheres do grupo com envelhecimento normal não participavam de nenhum grupo. De modo geral, as idosas podem ter tendência para o excesso ao informar o número de envolvimento do grupo em comparação com homens ${ }^{36}$. Além disso, as redes sociais dessas idosas são menos heterogêneas, constituídas essencialmente por familiares, filhos e netos ${ }^{37} \mathrm{e}$ associações religiosas $^{38}$.

Para entender melhor como a participação comunitária pode interferir positivamente no envelhecimento ativo e na melhoria da qualidade 
Tabela 3. Fatores associados ao envelhecimento ativo entre homens idosos residentes em comunidade. Sete lagoas-MG, 2012.

\begin{tabular}{|c|c|c|c|c|c|c|}
\hline \multirow{3}{*}{ Fatores determinantes } & \multicolumn{4}{|c|}{ Envelhecimento } & \multirow{3}{*}{$\begin{array}{c}\text { OR bruto } \\
\text { (IC95\%) }\end{array}$} & \multirow{3}{*}{ p-valor } \\
\hline & \multicolumn{2}{|c|}{ Normal $(\mathrm{N}=347)$} & \multicolumn{2}{|c|}{ Ativo $(\mathrm{N}=479)$} & & \\
\hline & $\mathbf{n}$ & $\%$ & $\mathbf{n}$ & $\%$ & & \\
\hline \multicolumn{7}{|l|}{ Qualidade de vida } \\
\hline Domínio Físico* & $57,01( \pm 16,17)$ & & $72,03( \pm 12,52)$ & & $1,07(1,05-1,08)$ & 0,000 \\
\hline Domínio Psicológico* & $64,53( \pm 15,11)$ & & $74,54( \pm 11,45)$ & & $1,03(1,01-1,05)$ & 0,001 \\
\hline Domínio Relações Sociais* & $66,19( \pm 17,16)$ & & $72,48( \pm 13,64)$ & & $0,99(0,98-1,01)$ & 0,231 \\
\hline Domínio Ambiental $^{*}$ & $58,58( \pm 12,97)$ & & $64,93( \pm 12,23)$ & & $1,02(1,00-1,04)$ & 0,032 \\
\hline Escore total ${ }^{*}$ & $63,51( \pm 11,39)$ & & $71,19( \pm 9,70)$ & & $1,03(1,01-1,05)$ & 0,008 \\
\hline \multicolumn{7}{|l|}{ Fatores transversais } \\
\hline \multicolumn{7}{|l|}{ Naturalidade } \\
\hline Sete Lagoas & 87 & 25,1 & 148 & 30,9 & $1,02(0,47-2,25)$ & 0,954 \\
\hline Outra cidade mineira & 248 & 71,5 & 313 & 65,3 & $0,80(0,37-1,71)$ & 0,565 \\
\hline Outro estado & 12 & 3,5 & 18 & 3,8 & 1 & \\
\hline \multicolumn{7}{|l|}{ Faixa etária } \\
\hline $60-74$ anos & 235 & 67,7 & 350 & 73,1 & $1,31(0,96-1,78)$ & 0,091 \\
\hline$\geq 75$ anos & 112 & 32,3 & 129 & 26,9 & 1 & \\
\hline \multicolumn{7}{|l|}{ Cor da pele } \\
\hline Branca & 115 & 33,2 & 183 & 38,7 & $1,29(0,94-1,76)$ & 0,117 \\
\hline Preta/Parda & 58 & 16,8 & 74 & 15,6 & $1,03(0,69-1,54)$ & 0,894 \\
\hline Outras & 173 & 50,0 & 216 & 45,7 & 1 & \\
\hline \multicolumn{7}{|l|}{ Estado civil } \\
\hline Casado & 253 & 72,9 & 362 & 75,6 & $1,11(0,80-1,54)$ & 0,531 \\
\hline Separado/solteiro/viúvo & 94 & 27,1 & 117 & 24,4 & 1 & \\
\hline \multicolumn{7}{|l|}{ Religião } \\
\hline Católica & 274 & 79,0 & 390 & 81,4 & $1,61(0,74-3,47)$ & 0,227 \\
\hline Evangélica & 46 & 13,3 & 69 & 14,4 & $1,72(0,74-4,03)$ & 0,207 \\
\hline Espírita & 08 & 2,3 & 05 & 1,0 & $0,75(0,19-2,92)$ & 0,682 \\
\hline Outra & 04 & 1,2 & 02 & 0,4 & $0,61(0,09-3,97)$ & 0,604 \\
\hline Sem religião & 15 & 4,3 & 13 & 2,7 & 1 & \\
\hline \multicolumn{7}{|c|}{ Fatores relacionados aos Sistemas de Saúde } \\
\hline \multicolumn{7}{|l|}{ Consulta médica } \\
\hline Sim & 308 & 88,8 & 392 & 81,8 & $0,56(0,38-0,85)$ & 0,006 \\
\hline Não & 39 & 11,2 & 87 & 18,2 & 1 & \\
\hline \multicolumn{7}{|l|}{ Plano de saúde } \\
\hline Sim & 205 & 59,1 & 261 & 54,5 & $0,73(0,52-1,03)$ & 0,069 \\
\hline Não & 142 & 40,9 & 218 & 45,5 & 1 & \\
\hline \multicolumn{7}{|l|}{ Serviço de saúde } \\
\hline Público & 199 & 57,3 & 278 & 58,0 & $1,19(0,77-1,84)$ & 0,436 \\
\hline Plano/Convênio & 97 & 28,0 & 145 & 30,3 & $1,53(0,95-2,46)$ & 0,081 \\
\hline Particular & 51 & 14,7 & 56 & 11,7 & 1 & \\
\hline
\end{tabular}

continua

de vida de idosos, seria imperativo investigar em longo prazo a densidade e a diversidade dessas associações, o nível de funcionamento democrático, e a extensão das conexões com outros grupos.

Entre os homens, os fatores comportamentais (não fumar, praticar atividade física e não ter relatado perda de apetite) foram preditores positivos para envelhecimento ativo.
Sedentarismo e tabagismo são importantes fatores de risco para a saúde e a qualidade de vida de idosos, amplamente discutidos na literatura ${ }^{30,39,40}$. Neste estudo, homens que praticavam alguma atividade física $(\mathrm{OR}=1,63 ; \mathrm{p}=0,008) \mathrm{e}$ que não fumavam $(\mathrm{OR}=0,53 ; \mathrm{p}=0,012)$ apresentaram maior probabilidade de ter envelhecimento ativo. No entanto, a atividade física e o hábito de fumar foram medidos usando uma única 
Tabela 3. continuação

\section{Envelhecimento}

Fatores determinantes

\begin{tabular}{|c|c|c|c|c|c|}
\hline \multicolumn{2}{|c|}{ Normal $(\mathrm{N}=347)$} & \multicolumn{2}{|c|}{ Ativo $(\mathrm{N}=479)$} & \multirow{2}{*}{$\begin{array}{c}\text { OR bruto } \\
\text { (IC95\%) }\end{array}$} & \multirow{2}{*}{ p-valor } \\
\hline n & $\%$ & $\mathbf{n}$ & $\%$ & & \\
\hline
\end{tabular}

Fatores comportamentais

Fumo

Sim
Não
Álcool
Sim
Não
Atividade física
Sim
Não
Medicamento
Sim
Não
Perda de apetite
Sim
Não
Edentulismo
Sim
Não

Fatores pessoais

Percepção de felicidade

Feliz

Nem feliz, nem infeliz Infeliz

Fatores relacionados ao ambiente físico Área

Urbana

Rural/distrito

$\begin{array}{cccccc}73 & 21,0 & 70 & 14,6 & 0,64(0,43-0,95) & 0,025 \\ 274 & 79,0 & 409 & 85,4 & 1 & \\ & & & & & \\ 111 & 32,0 & 146 & 30,5 & 0,90(0,65-1,25) & 0,544 \\ 236 & 68,0 & 333 & 69,5 & 1 & \\ & & & & & \\ 66 & 19,0 & 150 & 31,3 & 1,82(1,29-2,56) & 0,001 \\ 281 & 81,0 & 329 & 68,7 & 1 & \\ & & & & & \\ 268 & 77,2 & 311 & 64,9 & 0,57(0,41-0,80) & 0,001 \\ 79 & 22,8 & 168 & 35,1 & 1 & \\ & & & & & \\ 114 & 32,9 & 65 & 13,6 & 0,36(0,25-0,51) & 0,000 \\ 233 & 67,1 & 414 & 86,4 & 1 & \\ & & & & & \\ 227 & 65,6 & 293 & 61,2 & 0,92(0,68-1,25) & 0,594 \\ 119 & 34,4 & 186 & 38,8 & 1 & \\ & & & & & \\ & & & & & \\ 267 & 76,9 & 439 & 91,6 & 5,59(2,04-15,33) & 0,001 \\ 63 & 18,2 & 35 & 7,3 & 1,89(0,64-5,56) & 0,248 \\ 17 & 4,9 & 05 & 1,0 & & 1\end{array}$

Domicílio

Próprio

Alugado

De parentes

Outro

Esgoto canalizado

Sim

Não

Tipo de construção domicílio

Concreto

Outros

Abastecimento de água

Canalizado

Outros

Sanitário no domić́lio

Sim

Não

Coleta de lixo

Sim

Não

Eletricidade

Sim

Não

Quedas

Sim

\begin{tabular}{|c|c|c|c|c|c|}
\hline 309 & 89,0 & 415 & 86,6 & $0,88(0,46-1,68)$ & 0,700 \\
\hline 38 & 11,0 & 64 & 13,4 & 1 & \\
\hline 300 & 86,5 & 439 & 91,6 & $2,57(0,96-6,87)$ & 0,060 \\
\hline 14 & 4,0 & 19 & 4,0 & $2,44(0,74-8,08)$ & 0,143 \\
\hline 22 & 6,3 & 14 & 2,9 & $1,13(0,34-3,71)$ & 0,842 \\
\hline 11 & 3,2 & 07 & 1,5 & 1 & \\
\hline 324 & 93,4 & 437 & 91,2 & $0,95(0,40-2,27)$ & 0,914 \\
\hline 23 & 6,6 & 42 & 8,8 & 1 & \\
\hline 347 & 100,0 & 479 & 100,0 & $\ldots$ & $\ldots$ \\
\hline 0,0 & 0,0 & 0,0 & 0,0 & & \\
\hline 341 & 98,3 & 465 & 97,1 & $0,59(0,17-2,09)$ & 0,412 \\
\hline 6 & 1,7 & 14 & 2,9 & 1 & \\
\hline 345 & 99,4 & 473 & 98,7 & $0,37(0,04-3,38)$ & 0,375 \\
\hline 02 & 0,6 & 06 & 1,3 & 1 & \\
\hline 303 & 87,3 & 416 & 86,8 & $0,97(0,61-1,53)$ & 0,888 \\
\hline 44 & 12,7 & 63 & 13,2 & 1 & \\
\hline 346 & 99,7 & 479 & 100,0 & $0,0(0,00-0,00)$ & 1,000 \\
\hline 01 & 0,3 & 00 & 0,0 & 1 & \\
\hline 104 & 30,0 & 92 & 19,2 & $0,55(0,40-0,76)$ & 0,000 \\
\hline 243 & 70,0 & 387 & 80,8 & 1 & \\
\hline
\end{tabular}


Tabela 3. continuação

\begin{tabular}{|c|c|c|c|c|c|c|}
\hline \multirow{3}{*}{ Fatores determinantes } & \multicolumn{4}{|c|}{ Envelhecimento } & \multirow{3}{*}{$\begin{array}{c}\text { OR bruto } \\
\text { (IC95\%) }\end{array}$} & \multirow{3}{*}{ p-valor } \\
\hline & \multicolumn{2}{|c|}{ Normal $(\mathrm{N}=347)$} & \multicolumn{2}{|c|}{ Ativo $(\mathrm{N}=479)$} & & \\
\hline & $\mathbf{n}$ & $\%$ & $\mathbf{n}$ & $\%$ & & \\
\hline \multicolumn{7}{|c|}{ Fatores relacionados ao ambiente social } \\
\hline \multicolumn{7}{|c|}{ Escolaridade } \\
\hline Analfabeto & 107 & 30,8 & 133 & 27,8 & $0,43(0,25-0,73)$ & 0,002 \\
\hline 1 grau & 218 & 62,8 & 282 & 58,9 & $0,42(0,24-0,74)$ & 0,003 \\
\hline 2 grau & 22 & 6,3 & 64 & 13,4 & 1 & \\
\hline \multicolumn{7}{|l|}{ Arranjo domiciliar } \\
\hline Mora com cônjuge & 252 & 73,3 & 364 & 77,1 & $1,30(0,81-2,09)$ & 0,285 \\
\hline Arranjos mistos & 55 & 16,0 & 66 & 14,0 & $1,11(0,62-1,98)$ & 0,719 \\
\hline Mora sozinho & 37 & 10,8 & 42 & 8,9 & 1 & \\
\hline \multicolumn{7}{|l|}{ Participação em grupos } \\
\hline 0 & 280 & 80,7 & 382 & 79,7 & $0,96(0,67-1,39)$ & 0,833 \\
\hline$\geq 1$ & 67 & 19,3 & 97 & 20,3 & 1 & \\
\hline \multicolumn{7}{|l|}{ Amigos } \\
\hline 0 & 50 & 14,4 & 72 & 15,0 & $0,98(0,63-1,54)$ & 0,930 \\
\hline $1-10$ & 185 & 53,3 & 246 & 51,4 & $0,90(0,66-1,23)$ & 0,504 \\
\hline$\geq 11$ & 112 & 32,3 & 161 & 33,6 & 1 & \\
\hline \multicolumn{7}{|l|}{ Confiança } \\
\hline Sim & 44 & 12,7 & 67 & 14,0 & $1,07(0,70-1,65)$ & 0,749 \\
\hline Não & 303 & 87,3 & 412 & 86,0 & 1 & \\
\hline \multicolumn{7}{|l|}{ Percepção da segurança } \\
\hline Seguro & 156 & 45,0 & 241 & 50,3 & $1,24(0,90-1,70)$ & 0,196 \\
\hline Nem seguro, nem inseguro & 67 & 19,3 & 78 & 16,3 & $0,97(0,65-1,46)$ & 0,893 \\
\hline Inseguro & 124 & 35,7 & 160 & 33,4 & 1 & \\
\hline \multicolumn{7}{|l|}{ Fatores econômicos } \\
\hline \multicolumn{7}{|l|}{ Renda } \\
\hline$\leq \mathrm{R} \$ 622,00$ & 214 & 61,7 & 266 & 55,5 & $0,78(0,59-1,03)$ & 0,082 \\
\hline$>\mathrm{R} \$ 622,00$ & 133 & 38,3 & 213 & 44,5 & 1 & \\
\hline \multicolumn{7}{|l|}{ Aposentado } \\
\hline $\operatorname{Sim}$ & 305 & 87,9 & 394 & 82,3 & $0,64(0,43-0,95)$ & 0,029 \\
\hline Não & 42 & 12,1 & 85 & 17,7 & 1 & \\
\hline
\end{tabular}

*Valores expressados em média ( \pm desvio padrão).

pergunta com resposta dicotômica ( $\operatorname{sim} /$ não). Esta é uma limitação importante deste estudo, uma vez que estes resultados podem indicar que qualquer nível de atividade física estaria associado ao envelhecimento. Em relação ao tabagismo, seria importante investigar o tempo e a frequência do hábito no passado e atualmente.

O modelo feminino foi formado pelos fatores comportamentais, relacionados ao ambiente físico e social e a fatores econômicos. Mulheres com maior renda, que não sofreram quedas e com participação comunitária tiveram melhores escores de qualidade de vida e maior chance de pertencer ao grupo com envelhecimento ativo.

A prevalência de quedas entre mulheres foi alta $(37,6 \%)$, particularmente entre as idosas com mais de 75 anos (41,1\%). Este percentual é particularmente elevado em comparação com os dados do estudo SABE (Salud, Bienestar y Envejecimiento), que foi realizado em São Paulo com mulheres mais jovens $(33,0 \%)$ e mulheres com mais de 75 anos de idade $(36,9 \%)^{41}$. Um estudo qualitativo buscou explorar por que as mulheres mais velhas procuram ou não o médico depois de uma queda. Algumas mulheres não procuram ajuda porque acreditavam que sua queda ou lesão relacionada à queda não foi grave o suficiente para ir ao médico. Esses dados fornecem subsídios para destacar a importância do tema deste estudo, uma vez que essas prevalências poderiam ser bem maiores ${ }^{42}$. Nesse sentido, programas de prevenção de quedas podem melhorar a qualidade de vida de idosos ${ }^{43}$.

Finalmente, é importante discutir algumas limitações do estudo. Os resultados encontrados fazem parte da linha base do projeto AGEQOL. 
Tabela 4. Associações múltiplas entre envelhecimento ativo, qualidade de vida e fatores determinantes por gênero.

\begin{tabular}{|c|c|c|c|}
\hline Variáveis & $\begin{array}{c}\text { OR } \\
\text { ajustado }\end{array}$ & IC $95 \%$ & p-valor \\
\hline \multicolumn{4}{|l|}{ Masculino } \\
\hline Domínio Físico & 1,07 & $1,05-1,09$ & 0,000 \\
\hline Domínio Psicológico & 1,03 & $1,01-1,05$ & 0,022 \\
\hline Escore total & 1,03 & $1,01-1,05$ & 0,021 \\
\hline \multicolumn{4}{|l|}{ Fumo } \\
\hline Sim & 0,53 & $0,34-0,82$ & 0,012 \\
\hline Não & 1 & & \\
\hline \multicolumn{4}{|l|}{ Atividade física } \\
\hline Sim & 1,63 & $1,09-2,44$ & 0,008 \\
\hline Não & 1 & & \\
\hline \multicolumn{4}{|l|}{ Perda de apetite } \\
\hline Sim & 0,53 & $0,35-0,80$ & 0,003 \\
\hline Não & 1 & & \\
\hline \multicolumn{4}{|l|}{ Participação em grupos } \\
\hline$\geq 1$ & 1,88 & $1,21-2,93$ & 0,005 \\
\hline 0 & 1 & & \\
\hline \multicolumn{4}{|l|}{ Aposentado } \\
\hline $\operatorname{Sim}$ & 0,48 & $0,29-0,79$ & 0,004 \\
\hline Não & 1 & & \\
\hline \multicolumn{4}{|l|}{ Feminino } \\
\hline Domínio Físico & 1,05 & $1,03-1,06$ & 0,000 \\
\hline Domínio Psicológico & 1,01 & $1,00-1,03$ & 0,040 \\
\hline Escore total & 1,02 & $1,01-1,04$ & 0,002 \\
\hline \multicolumn{4}{|l|}{ Perda de apetite } \\
\hline Sim & 0,66 & $0,49-0,89$ & 0,006 \\
\hline Não & 1 & & \\
\hline \multicolumn{4}{|l|}{ Quedas } \\
\hline Sim & 0,64 & $0,49-0,84$ & 0,001 \\
\hline Não & 1 & & \\
\hline \multicolumn{4}{|l|}{ Participação em grupos } \\
\hline$\geq 1$ & 1,65 & $1,20-2,26$ & 0,002 \\
\hline 0 & 1 & & \\
\hline \multicolumn{4}{|l|}{ Renda } \\
\hline$\leq \mathrm{R} \$ 622,00$ & 0,66 & $0,50-0,80$ & 0,006 \\
\hline$>\mathrm{R} \$ 622,00$ & 1 & & \\
\hline
\end{tabular}

Por isso, ainda não é possível determinar se existe uma relação temporal entre envelhecimento ativo, qualidade de vida e as demais variáveis analisadas.

Apesar da iniciativa brasileira de propor um instrumento para mensurar o envelhecimento ativo $^{20,21}$, ainda não há na literatura instrumentos validados. Não há consenso entre os autores sobre a melhor forma de realizar esta análise. $\mathrm{Na}$ literatura internacional, encontrou-se um mo- delo comunitário de prevenção de doenças crônicas para envelhecimento ativo desenvolvido e implantado no México ${ }^{44}$ e uma escala de envelhecimento ativo para adultos tailandeses (Scale of Active Aging for Thai Adults - AAS-Thai $)^{45}$. Bowling ${ }^{30}$ tem investigado o envelhecimento ativo por meio de duas perguntas: "O que, em sua opinião, são as coisas associadas a 'envelhecimento ativo'?" e "Pensando nas coisas que você listou como associada ao envelhecimento ativo, você diria que você está envelhecendo 'ativamente' até agora?".

Este estudo é um dos primeiros a propor um método específico de análise para medir o envelhecimento ativo em uma amostra de idosos brasileiros. Utilizou-se a análise de segmentação e discriminante canônica para compilar os testes de rastreio usados em pesquisas com idosos (capacidade funcional, capacidade cognitiva, percepção de saúde, sintomas depressivos e funcionalidade familiar) em uma medida única para envelhecimento ativo. Esta análise foi efetuada para criar dois grupos internamente mais homogêneos. Encontramos classificação correta de $100 \%$ e alto coeficiente de correlação $(0,90)$, indicando que a medida de envelhecimento ativo para os idosos desta amostra é estaticamente confiável ( $\mathrm{p}=0,000)$.

Este estudo é um dos poucos realizados com uma amostra probabilística de idosos vivendo em comunidade sem déficits cognitivos e/ou físicos significativos, com número suficiente de participantes para realizar uma análise estatística separada por gênero. Esta amostra corresponde a $10 \%$ de todos os idosos do município.

Conclui-se que qualidade de vida e a participação em grupos são os principais determinantes de envelhecimento ativo, e que os demais fatores associados são diferentes para cada gênero. Sugere-se que futuras pesquisas englobem outros testes de força, mobilidade e equilíbrio na construção de uma escala psicométrica que possibilite medir ainda melhor o nível de envelhecimento ativo entre idosos vivendo em comunidade. Além disso, seria interessante incluir mais variáveis relacionadas aos aspectos culturais (etnia, origem, religiosidade e espiritualidade) e aspectos pessoais (imagem corporal, sexualidade, fatores genéticos e psicológicos).

Nesse contexto, espera-se que as diferenças de gênero sejam ainda mais marcantes na avaliação dos determinantes do envelhecimento ativo na vida dos idosos e sua repercussão em sua qualidade de vida. 


\section{Colaboradores}

AC Campos participou da concepção, análise e interpretação dos dados e redação do artigo. AMD Vargas e EF Ferreira participaram participaram da concepção, interpretação dos dados e revisão crítica do artigo. Todos os autores aprovaram a versão final do artigo.

\section{Agradecimentos}

À CAPES, ao CNPq e à FAPEMIG pelo financiamento.

\section{Referências}

1. Organização Mundial da Saúde (OMS). Envelhecimento ativo: uma politica de saúde. World Health Organization. Brasília: Organização Pan-Americana da Saúde (OPAS); 2005. [acessado 2010 maio 28]. Disponível em: http://dtr2001.saude.gov.br/svs/pub/pdfs/envelhecimento_ativo.pdf.

2. Silva HS, Lima AMM, Galhardoni, R. Envelhecimento bem-sucedido e vulnerabilidade em saúde: aproximações e perspectiva. Interface (Botucatu) 2010; 14(35):867-877.

3. The World Health Organization quality of life assessment (WHOQOL): position paper from the World Health Organization. Soc Sci Med 1995; 41(10):1403-1410.

4. Martins JJ, Schneider DG, Coelho FL, Nascimento ERP, Albuquerque GL, Erdmann AL, Gama FO. Avaliação da qualidade de vida de idosos que recebem cuidados domiciliares. Acta Paul Enferm 2009; 22(3):265-271.

5. Lowsky DJ, Olshansky SJ, Bhattacharya J, Goldman DP. Heterogeneity in healthy aging. J Gerontol A Biol Sci Med Sci 2014; 69(11):640-649.

6. Hekmatpou D, Shamsi M, Zamani M. The effect of a healthy lifestyle program on the elderly's health in Arak. Indian J Med Sci 2013; 67(3-4):70-77.

7. De Luca d'Alessandro E, Bonacci S, Giraldi G. Aging populations: the health and quality of life of the elderly. Clin Ter 2011; 162(1):e13-e18.

8. Sete Lagoas. Lei no 7804, de 14 de outubro de 2009. Define as Regiões Administrativas - RA no perímetro urbano do distrito sede do município de Sete Lagoas. [acessado 2011 abril 07]. Disponível em: http://www.jusbrasil. com.br/legislacao/834407/lei-7804-09-sete-lagoas.

9. Instituto Brasileiro de Geografia e Estatística (IBGE). Índice Provisório População 2011. Censo Demográfico 2010. [acessado 2011 abril 07]. Disponível em: http:// www.ibge.gov.br/home/estatistica/populacao/censo 2010/MG2010.pdf.

10. Santos JLF, Lebrão ML, Duarte YAO, Lima FD. Functional performance of the elderly in instrumental activities of daily living: an analysis in the municipality of São Paulo, Brazil. Cad Saude Publica 2008; 24(4):879886.

11. Instituto Brasileiro de Geografia e Estatística (IBGE). Ficha da PNAD 2011. [acessado 2011 abril 07]. Disponível em: ftp://ftp.ibge.gov.br/Trabalho_e_Rendimento/Pesquisa_Nacional_por_Amostra_de_Domicilios_ anual/2011/q_pnad2011.pdf.

12. World Health Organization (WHO). Oral health surveys: basic methods. $4^{\text {th }}$ Ed. Geneva: WHO; 1997. Disponível em: http://www2.paho.org/hq/dmdocuments/2009/OH_st_Esurv.pdf.

13. Brucki SMD, Nitrini R, Caramelli P, Bertolucci PHF, Okamoto IH. Sugestões para o uso do mini-exame do estado mental no Brasil. Arq Neuropsiquiatr 2003; 61(3B):77-81.

14. Quiroga P, Albala C, Klaasen. Validación de un test de tamizaje para el diagnóstico de demencia asociada a edad, en Chile. Rev Med Chil 2004; 132(4):467-478.

15. Almeida OP, Almeida AS. Confiabilidade da versão brasileira da Escala de Depressão em Geriatria (GDS) versão reduzida. Arq Neuropsiquiatr 1999; 57(2B):421426. 
16. Duarte YAO, Lebrão ML, Lima FD. Contribuição dos arranjos domiciliares para o suprimento de demandas assistenciais dos idosos com comprometimento funcional em São Paulo, Brasil. Pan Am J Public Health 2005; 17(5/6):370-378.

17. Hair JF, Black WC, Babin JB, Anderson RE, Tatham RL. Multivariate Data Analysis. New Jersey: PrenticeHall; 2006.

18. Fleck MPA, Louzada S, Xavier M, Chachamovich E, Vieira G, Santos L, Pinzon V. Application of the Portuguese version of the abbreviated instrument of quality life WHOQoL-bref. Rev Saude Publica 2000; 34(2):178183.

19. Fleck MPA, Chachamovich E, Trentini CM: Development of the WHOQoL-old module in Brazil. Rev Saude Publica 2006; 40(5):785-791.

20. Farias RG, Santos SMA. Influência dos determinantes do envelhecimento ativo entre idosos mais idosos. Texto Contexto Enferm 2012; 21(1):167-176.

21. Vicente FR, Santos SMA. Avaliação multidimensional dos determinantes do envelhecimento ativo em idosos de um município de Santa Catarina. Texto Contexto Enferm 2013; 22(2):370-378.

22. Hosmer DW, Lemeshow S. Applied logistic regression. $2^{\text {th }}$ ed. New York: John Wiley and Sons Inc; 1989.

23. Pregibon D. Logistic Regression Diagnostics. Mineapolis: Annals of Statistic; 1981.

24. Oliveira SES, Hohendorff JV, Müller JL, Bandeira DR, Koller SH, Fleck MPA, Trentini CM. Associations between self-perceived quality of life and socio-demographic, psychosocial, and health variables in a group of elderly. Cad Saude Publica 2013; 29(7):1437-1448.

25. Trentini CM, Chachamovich E, Wagner GP, Müller DH, Hirakata VN, Fleck MPA. Quality of life (QoL) in a Brazilian sample of older adults: the role of sociodemographic variables and depression symptoms. Appl Res Qual Life 2011; 6(3):291-309.

26. Alexandre TS, Cordeiro RC, Ramos LR. Factors associated to quality of life in active elderly. Rev Saude Publica 2009; 43(4):613-621.

27. Bowling A, Banister D, Sutton S, Evans O, Windsor J. A multidimensional model of the quality of life in older age. Aging Ment Health 2002; 6(4):355-371.

28. Fernandes MGM. Papéis sociais de gênero na velhice: $\mathrm{o}$ olhar de si e do outro. Rev Bras Enferm 2009; 62 (5):705710

29. Figueiredo MLF, Tyrrel MA, Carvalho CMRG, Luz MHBA, Amorim FCM, Loiola NLA. As diferenças de gênero na velhice. Rev Bras Enferm 2007; 60(4):422427.

30. Bowling A. Perceptions of active ageing in Britain: divergences between minority ethnic and whole population samples. Age Ageing 2009; 38(6):703-710.

31. Moraes JF, Azevedo e Souza VB. Factors associated with the successful aging of the socially-active elderly in the metropolitan region of Porto Alegre. Rev Bras Psiquiat 2005; 27(4):302-308.
32. Figueira HA, Giani TS, Beresford H, Ferreira MA, Mello D, Figueira AA, Figueira JA, Dantas EH. Quality of life (QOL) axiological profile of the elderly population served by the Family Health Program (FHP) in Brazil. Arch Gerontol Geriatr. 2009; 49(3):368-372.

33. Kirchengast S, Haslinger B. Gender differences in health-related quality of life among healthy aged and old-aged Austrians: cross-sectional analysis. Gend Med 2008; 5(3):270-278

34. Cherepanov D, Palta M, Fryback DG, Robert SA, Hays RD, Kaplan RM. Gender differences in multiple underlying dimensions of health-related quality of life are associated with sociodemographic and socioeconomic status. Med Care 2011; 49(11):1021-1030.

35. Kishimoto Y, Suzuki E, Iwase T, Doi H, Takao S. Group involvement and self-rated health among the Japanese elderly: an examination of bonding and bridging social capital. BMC Public Health 2013; 13:1189.

36. Camarano AM. Os Novos Idosos Brasileiros: Muito Além dos 60? Rio de Janeiro: IPEA; 2004.

37. Gontijo EEL, Gontijo M, Lourenço AFE, Inocente NJ. O capital social de idosos atendidos no ambulatório do Centro Universitário UNIRG na cidade de Gurupi, Tocantins. Saud. Pesq. 2013; 6(1):43-51.

38. Geib LLTC. Determinantes sociais da saúde do idoso. Cien Saude Colet 2012; 17(1):123-133.

39. Ribeiro PCC, Neri AL, Cupertino APFB, Yassuda MS. Variabilidade no envelhecimento ativo segundo sexo, idade e saúde. Psicol. estud. 2009; 14(3):501-509.

40. Ferreira OGL, Maciel SC, Costa SMG, Silva AO, Moreira MASP. Envelhecimento ativo e sua relação com a independência funcional. Texto Contexto Enferm 2012; 21(3):513-518.

41. Lebrão ML, Laurenti R. Saúde, bem-estar e envelhecimento: o estudo SABE no Município de São Paulo. Rev Bras Epidemiol 2005; 8(2):127-141.

42. Dollard J, Braunack-Mayer A, Horton K, Vanlint S. Why older women do or do not seek help from the GP after a fall: a qualitative study. Fam Pract 2014; 31(2):222-228.

43. Nicolussi AC, Fhon JRS, Santos CAV, Kusumota L, Marques S, Rodrigues RAP. Qualidade de vida em idosos que sofreram quedas: revisão integrativa da literatura. Cien Saude Colet 2012; 17(3):723-730.

44. Mendoza-Núñez VM, Martínez-Maldonado ML, Correa-Muñoz E. Implementation of an active aging model in Mexico for prevention and control of chronic diseases in the elderly. BMC Geriatr 2009; 9:40.

45. Thanakwang K, Isaramalai SA, Hatthakit U. Development and psychometric testing of the active aging scale for Thai adults. Clin Interv Aging. 2014; 9:1211-1221.

Artigo apresentado em 16/09/2014

Aprovado em 13/11/2014

Versão final apresentada em 15/11/2014 